\title{
TOMMASO CAMPANELLA E L'ETERNITÀ \\ DEL MONDO
}

\section{TOMMASO CAMPANELLA AND THE ETERNITY OF THE WORLD}

\author{
RICCARDO CHIARADONNA \\ Università degli studi Roma Tre"
}

Resumen: El presente estudio examina la discusión sobre la eternidad del mundo en Tommaso Campanella, desde la Philosophia sensibus demonstrata, publicada en 1591 en defensa de Telesio contra las críticas del aristotélico Giacomo Antonio Marta, hasta la Metaphysica, escrita por Campanella in varias redacciones y publicada finalmente en París en 1638. La posición de Campanella presenta algunos importantes aspectos, constantes a lo largo de toda su reflexión filosofica, especialmente la crítica del eternalismo aristotélico y el vínculo con la tradición platónico-ficiniana de la prisca theologia. A este planteamiento, cuyos orígenes deben llevarse a la antigua tradición de debates acerca de Platón y Aristóteles, proseguida hasta el siglo XV, se añade en las obras más maduras la apelación a la nueva astronomia, que, como subraya Campanella, ha venido a dar el golpe mortal definitivo a la cosmología aristotélica. Por otra parte, la Apologia pro Galileo (1616) demuestra que Campanella trató de insertar la astronomía misma de Galileo en el cuadro de la prisca theologia.

Palabras clave: Tommaso Campanella, eternidad del mundo, cosmología, Aristóteles y aristotelismo, prisca theologia, Marsilio Ficino

Aвstract: This study examines Tommaso Campanella's discussion of the eternity of the world, as presented in texts ranging from Philosophia sensibus demonstratapublished to defend Telesio from Giacomo Antonio Marta's criticism, in 1591 - to Metaphysica, which Campanella drafted in a number of different versions and finally published in Paris in 1638. Campanella's stance is marked by some striking recurrent features: most notably, his criticism of Aristotelian eternalism and a connection with

" Professore associato di Storia della filosofia antica. Via Ostiense, 234-00146 Roma. riccardo. chiaradonna@uniroma3.it. 
the Platonic-Ficinian tradition of prisca theologia. This framework, stemming from the ancient tradition of debates on Plato and Aristotle that extended well into the $15^{\text {th }}$ century, is combined in Campanella's later works with a reference to the new astronomy, which —as the philosopher himself stresses - delivered a final blow to Aristotelian cosmology. The Apologia pro Galileo (1616) further shows that Campanella sought to incorporate Galileo's astronomy into the framework of prisca theologia.

KeYwords: Tommaso Campanella, eternity of the world, cosmology, Aristotle and Aristotelian tradition, prisca theologia, Marsilius Ficinus.

\section{La Philosophia sensibus demonstrata e polemica antiperipatetica}

Il tema dell'eternità del mondo è costantemente presente nell'opera di Tommaso Campanella ed è indissolubilmente collegato alla polemica contro Aristotele. Nel presente contributo mi propongo di illustrare la posizione antiaristotelica di Campanella inquadrandola nei dibattiti che, dall'antichità in poi, si sono svolti circa la generazione e l'eternità del cosmo, dibattiti suscitati principalmente dal Timeo di Platone e dalla critica antiplatonica nel De caelo di Aristotele.

Particolare rilievo ha la Philosophia sensibus demonstrata, scritta da Campanella a 21 anni e pubblicata a Napoli nel $1591^{1}$. L'opera è divisa in otto "dispute» (disputationes) nelle quali Campanella difende la filosofia naturalistica di Telesio contro gli attacchi dell'aristotelico Giacomo Antonio Marta. Si tratta di un'opera centrale per comprendere il percorso filosofico di Campanella. Certamente è uno scritto giovanile, in cui è prevalente l'adesione al pensiero di Telesio e non emergono i temi della matura riflessione filosofica di Campanella (in particolare la sua elaborata metafisica). Sarebbe però molto riduttivo considerare la Philosophia sensibus demonstrata solo come testimonianza di una fase precoce e poco matura, destinata a essere superata dalle opere posteriori. È più fruttuoso leggere il lavoro giovanile come una mappa dei principali temi che si ritroveranno pienamente sviluppati nelle opere successive ${ }^{2}$. C'è, inoltre, un altro aspetto di grande

1 Per maggiori dettagli, si veda Firpo (1940), p. 31. Si cita qui l'edizione curata da Luigi De Franco: cfr. Campanella (1992), che contiene un dettagliato apparato di fonti. Un'ottima discussione sintetica sull'opera e sulla presenza del pensiero di Telesio in Campanella si trova in Ernst (2002), pp. 8-16; si veda anche Granada (2007), p. 281-282.

2 In questa prospettiva, condividerei la valutazione recentemente proposta da GATTI (2010), pp. 31-76 circa la sostanziale continuità del pensiero di Campanella tra l'opera giovanile e i lavori della maturità. Condivisibile mi pare anche il parallelo stabilito dalla studiosa tra la Philosophia 
importanza. Il giovane Campanella aveva assimilato, sorretto da una memoria eccezionale, una quantità imponente di letture. Almeno in parte, le fonti che confluiscono nella Philosophia sensibus demonstrata restano costanti nelle opere successive, che tuttavia (anche perché spesso composte in carcere) sono talvolta meno precise nelle indicazioni ${ }^{3}$. Per questa ragione, l'opera giovanile non soltanto permette di delineare una mappa precoce dei temi filosofici principali di Campanella, ma consente di tracciare con buona precisione una sorta di biblioteca campanelliana e di individuare i testi che costituiscono maggiore fonte di ispirazione per questo autore.

Nel contrapporsi ad Aristotele, Campanella segue la traccia stabilita da Telesio nel De rerum natura. Significativamente, Campanella pone in rilievo l'accordo del pensiero di Telesio con la rivelazione biblica: «Nos autem dicimus quod Telesii doctrina in nullis a Moyse discordat $»^{4}$. Sono invece Aristotele e i Peripatetici a collocarsi fuori di essa, tanto da essere definiti lapidariamente, fin dalla Praefatio, come «eretici» ${ }^{5}$. Proprio riguardo alla creazione Campanella ha buon gioco a contestare l'argomentazione di Marta contro Telesio. L'errore è duplice: da un lato, Marta occulta che proprio Aristotele non ha ammesso la creazione; dall'altro, tace sulla critica che Telesio ha mosso contro la dottrina aristotelica dell'eternità del mondo 6 .

sensibus demonstrata e la critica antiaristotelica del successivo De gentilismo non retinendo, redatto nel 1609 e pubblicato a Parigi nel 1636: cfr Campanella (1636). Meno persuasiva mi sembra invece la lettura generale sviluppata da Gatti, la quale si propone di respingere ogni interpretazione di tipo naturalistico mostrando come Campanella sia stato, in tutte le fasi del suo pensiero, in linea con le istanze del cattolicesimo romano: cfr., ad esempio, Gatti (2010), p. 7. Più equilibrato e convincente il giudizio di Granada (2007), p. 282 che, sottolineando il punti di distanza della Philosophia sensibus demonstrata rispetto a Telesio, osserva: «... Campanella abandons Telesio's exclusively naturalist perspective to root physiologia in a metaphysical and theological discourse that basically hearkens back to Platonism, and especially to Campanella's reading of Ficino. This need to root physics in higher principles that ultimately connect back to God would be a permanent feature of Campanella's thought and would condition - in accordance with his conception of the relationship between Divine Providence and the process of nature- his future attitude towards Galileo's new science».

3 Cfr. Chiaradonna (2010), p. 329.

4 Campanella (1992), p. 522.

5 Campanella (1992), p. 15: «At puri Peripatetici sunt haeretici, ut patuit».

6 Campanella (1992), pp. 125-126. «Sed Marta deinde longe rudem promit sermonem, dum dicit Telesium non agnoscere creationem; ipse enim Aristotelem non ponentem creationem ubique damnat et praecipue in 4 libro, capitibus 27 et 29, et in 1 maioris, capite 1 , et 2 minoris circa finem». 
La prima Disputatio (De principiis rerum naturalium) comincia con una netta affermazione del carattere generato del cosmo rispetto a un principio produttore. Aristotele è contrapposto all'insieme dei filosofi che hanno colto questa fondamentale verità: "Huic universorum machinae, quam mundum appellamus, principium aliquod primo productivum philosophantes omnes vel quidam modo praeter Aristotelem assignavere» ${ }^{7}$. La polemica prosegue a lungo e molte sezioni dell'opera sono, più o meno direttamente, riconducibili alla questione dell'eternità del cosmo. Tuttavia, è la prima parte della terza Disputatio a fornire il materiale più interessante. L'argomento è De coelo et mundo. Gran parte della discussione è dedicata alla confutazione della tesi aristotelica sulla natura dei corpi celesti, dei quali Campanella, seguendo Telesio, afferma la composizione ignea. La prima parte della trattazione è però espressamente dedicata al tema dell'eternità del mondo e la confutazione di Aristotele si unisce a un'ampia discussione delle autorità antiche. Ad essere ricordato per primo è Anassagora, che pose come principio materiale della realtà il chaos in cui tutte le cose erano celate, e dal quale l'intelletto le separò ordinando il cosmo. Certamente erano note a Campanella le opere di Platone e Aristotele, nelle quali si illustrano le tesi di Anassagora ${ }^{8}$. Tuttavia, in questo passo Campanella si fonda piuttosto sul Liber de historia philosophica attribuito a Galeno (cap. 35 De Deo), dal quale provengono anche le informazioni sull'epicureo Metrodoro menzionato poco sotto?.

Le autorità inizialmente richiamate da Campanella a sostegno della generazione del cosmo dal chaos primigenio sono Anassagora, Esiodo, Museo, Orfeo e Ovidio. Essi sono inoltre giudicati concordi con la rivelazione biblica, come Campanella afferma fondandosi sull'autorità di Pico: «hanc sententiam Picus Mirandulanus allegorizat et concordat cum ea Moysi, qui etiam chaos in principio ponit» ${ }^{10}$. L'eredità dell'insegnamento ficiniano e della prisca theologia è qui molto chiara. Alle opinioni sulla generazione del cosmo dal chaos, Campanella fa seguire le tesi di Empedocle ed Eraclito, che pongono generazione e distruzione cicliche, seguite dalle dottrine degli atomisti Democrito e Metrodoro. Viene poi la tesi della generazione del mondo da Dio sostenuta da «uomini divinissimi», tra cui Platone:

7 Campanella (1992), p. 25.

8 Cfr., in particolare, Platone, Phaedo, 97c-e; Aristotele, Metaphysica, I, 3, 984c11-16; I, 4, 985a18-21. Si veda, sulla sezione del Fedone dedicata ad Anassagora, Campanella (1992), p. 47.

9 Testo greco in Diels (1879), pp. 617-618. Lopera era stata tradotta in latino da Giulio Marziano Rota (con pesanti alterazioni) e pubblicata nella prima edizione giuntina di Galeno nel 1541-1542; quindi più volte ristampata. Sulle vicende editoriali di questo opuscolo e la sua attribuzione a Galeno, cfr. Mansfeld in Mansfeld-Runia (1997), pp. 21-28.

10 Campanella (1992), p. 285. 
Divinissimi autem viri, Pythagoras Samius, Calabrorum doctor, Plato Atheniensis, Mercurius Trismegistus Aegyptius, et Calaber Timaeus Locrensis, mundum a Deo optimo maximo emanasse enuntiant aperte ac interire posse; Dei tamen voluntate aevitermum, sicut et Socrates aiebat ${ }^{11}$.

Si ha dunque la dottrina del Timeo di Platone ${ }^{12}$, inserita in una tradizione più ampia che comprende Pitagora, Mercurio Trismegisto, il pitagorico Timeo di Locri.

\section{L'eredità degli Antichi: esegesi, commenti e prisca theologia}

Come si è già notato, nei riferimenti di Campanella sono ben riconoscibili i contorni della sistemazione operata da Marsilio Ficino e della prisca theologia ${ }^{13}$. Sono però necessarie alcune precisazioni supplementari. Gli specialisti hanno giustamente considerato la polemica di Campanella contro Aristotele, e le sue idee sulla tradizione filosofica, come l'ultimo capitolo nella controversia su Platone e Aristotele che oppose nel Rinascimento sostenitori e detrattori di Aristotele, platonici umanisti e filosofi averroisti. L'origine della controversia si deve situare nell'opera di Gemisto Pletone De differentiis Aristotelis et Platonis (1439) ${ }^{14}$. Essa continuò nella polemica tra Giorgio Trapezunzio e il Cardinale Bessarione, prima di confluire in Ficino e, quindi, negli autori cinquecenteschi ${ }^{15}$. Campanella giunge alla fine di questo dibattito: la sua Metaphysica è pubblicata nel 1638, circa due secoli dopo il soggiorno di Pletone a Firenze. Tutto ciò è indubbiamente vero. D'altra parte, è importante sottolineare che sia il dibattito su Aristotele, sia la

11 Campanella (1992), p. 286. Circa le fonti di Campanella in questa sezione (principalmente, la traduzione ermetica di Ficino e il Compendium in Timaeum dello stesso Ficino, si vedano le note ad loc. di De Franco. Cfr., in particolare, Ficino (1576), p. 1443 (Compendium In Timaeum, XIII) sulla dossografia circa la generazione del cosmo. Sul commento ficiniano del Timeo e il suo impatto nella cultura del Cinquecento fino a Galileo, cfr. Hankins (2000), pp. 413-416. Della traduzione ermetica esiste una recente edizione critica: cfr. Ficino (2011).

12 Cfr. Plato, Timaeus, 28b, 41b.

${ }^{13} \mathrm{Vi}$ è in proposito un'abbondante letteratura critica. Mi limito a rinviare, sul programma ficiniano e la sua genesi, al recente contributo di Gentile (2012).

${ }_{14} \mathrm{Si}$ veda il contributo di Miguel Ángel Granada in questo volume.

15 Per ulteriori dettagli, cfr. Hankins (1990), pp. 165-263, Mahoney (2003), Del Soldato (2012); sulla posizione di Ficino, cfr. Monfasani (2002). Sulle polemiche quattrocentesche intorno a Platone e il loro significato politico, restano fondamentali le pagine di Garin (1975), pp. 113-120. Rinvio, infine, alla recente traduzione italiana dell'Adversus calumniatorem Platonis: Bessarione (2013). Sulla posterità cinquecentesca della controversia, cfr. Muccillo (2012). 
dottrina dell'unità delle tradizioni teologiche che fa da base alla prisca theologia, hanno origini molto più antiche.

Si consideri, in primo luogo, il richiamo di Campanella a Timeo di Locri. Lo scritto De natura mundi et animae (Peri physios kosmô kai psychas), attribuito a questo autore, è un apocrifo, la cui composizione va fissata probabilmente intorno al I secolo a.C. Si tratta di una parafrasi del Timeo destinata a dimostrare che le dottrine formulate da Platone sono in realtà più antiche e hanno una paternità pitagorica ${ }^{16}$. Campanella segue questa tesi tradizionale: «Timaeus vero, conterraneus noster, eadem dixit quae Plato, eius dicipulus» ${ }^{17}$. L'origine di una simile tradizione, lontanissima antenata della prisca theologia, può farsi risalire al periodo tra I secolo a.C. e II secolo d.C. Fu allora, dopo la fine dell'Accademia platonica scettica di età ellenistica, che il rinnovato interesse per il platonismo metafisico e cosmologico condusse autori di ispirazione platonica (in particolare Eudoro di Alessandria e il suo circolo) a richiamarsi all'autorità di Pitagora stabilendo così una precisa genealogia filosofica che legava Platone a maestri più antichi. Probabilmente questa posizione arcaizzante era determinata dall'opposizione verso le scuole ellenistiche (Stoici ed Epicurei), che si erano poste in aperta contrapposizione rispetto ai maestri del IV secolo a.C. Dal I secolo a.C., dunque, i filosofi platonici (ma anche quelli aristotelici) cominciano a richiamarsi sistematicamente agli «antichi maestri» (veteres, palaioi) trovando nelle loro dottrine garanzia di verità ${ }^{18}$. Vi erano anche altre ragioni per questa svolta arcaizzante. In particolare, dopo la fine delle scuole filosofiche ateniesi all'inizio del I secolo a.C. (e più precisamente intorno all' 86 a.C., a seguito del saccheggio di Silla nella prima guerra mitridatica), per i circoli filosofici sparsi nei centri culturali del Mediterraneo affermare un'affiliazione filosofica implicava basarsi sui testi dei loro ideali maestri: era infatti ormai diventato impossibile fondare la propria identità dottrinale su una continuità istituzionale rispetto ai fondatori delle scuole. Fu così che l'elaborazione filosofica cominciò a collegarsi indissolubilmente all'esegesi e al commento di testi normativi. Anche se la filosofia antica è legata all'esegesi fin dalle origini, è nel I secolo a.C. che comincia l'età del commento filosofico, connessa a una concezione arcaizzante della filo-

16 Sullo scritto, si veda Baltes (1972). Uno status quaestionis sulla genesi degli apocrifici pitagorici si trova in Centrone (1990), pp. 14-15. Circa la ricezione di Timeo di Locri in Ficino, si veda Hankins (2000), p. 214.

17 Campanella (1992), p. 47.

${ }^{18}$ Cfr. Frede (1999), pp. 782-785, 788. 
sofia: circa 1700 anni dopo, Campanella segna la fine di questo peculiare modo di concepire la filosofia ${ }^{19}$.

Come si è prima accennato, particolare importanza ha, all'inizio di questo processo, Eudoro di Alessandria, enigmatica figura di platonico pitagorizzante vissuto probabilmente nella seconda metà del I secolo a.C., alla cui influenza va probabilmente ricondotta la composizione di alcuni scritti pitagorici apocrifi ${ }^{20}$. Il tema dell'unità delle tradizioni teologiche diventerà capitale nel neoplatonismo, soprattutto da Porfirio in poi e, in modo del tutto centrale, in Siriano e Proclo, gli scolarchi della scuola neoplatonica di Atene nel V secolo d.C. ${ }^{21}$. Probabilmente, i loro sforzi per dimostrare l'unità della tradizione teologica ellenica (nella Theologia Platonica, Proclo traccia una linea di successione che parte da Orfeo, il primo dei teologici greci, e passa ad Aglaofemo, Pitagora, Filolao e Platone), possono essere compresi appieno solo tenendo conto della loro posizione anticristiana ${ }^{22}$. Mostrare l'unità delle tradizioni teologiche elleniche permetteva infatti ai neoplatonici di stabilire una sorta di fronte comune contro il cristianesimo. In questo modo, però, essi finivano per costruire un'unitaria lettura della filosofia greca, di tipo teologizzante, che, attraverso numerose mediazioni, si sarebbe rivelata particolarmente favorevole proprio per essere assimilata dalla filosofia cristiana dei secoli posteriori.

Possiamo sintetizzare così ciò che è stato appena illustrato sommariamente. La tesi secondo cui vi sarebbe una profonda unità tra le tradizioni filosofiche e teologiche antiche, secondo una linea di successione che comprende sia Pitagora sia Platone, può addirittura farsi risalire alla rinascita del platonismo dogmatico nel I secolo a.C. (quello che viene chiamato, nel dibattito erudito, «medioplatonismo»). Questa idea ebbe vita tanto lunga quanto complessa e, in particolare, è

19 Per maggiori dettagli circa il I secolo a.C. rinvio ad alcuni fondamentali di riferimento: Hadot (1987), Donini (1994), Sedley (2003). Si vedano, inoltre, due recenti raccolte di articoli: Bonazzi e Opsomer (2009), Schofield (2013).

${ }^{20}$ In realtà, è Antioco di Ascalona il primo filosofo accademico a rivendicare il ritorno agli «antichi» contro la posizione dell'Accademia scettica. Tuttavia, nei frammenti pervenuti a noi non vi è traccia di una posizione pitagorizzante e Antioco sembra piuttosto aver voluto incorporare, con un ruolo comunque subordinato al platonismo, concezioni stoiche. Si vedano, adesso, i contributi raccolti in SEDLEY (2012).

${ }^{21}$ Si veda SAFfrey (1992) e, per un'aggiornata presentazione sintetica, TAormina (2012).

${ }^{22}$ Cfr. Proclo, Theologia platonica, I, 5, 25, 24-26, 4 Saffrey - Westerink. La genealogia di Proclo è ripresa da Marsilio Ficino nell'Argumentum premesso al Pimander, con l'aggiunta di Ermete Trismegisto: cfr. Ficino (1576), p. 1879 = Ficino (2011), p. 4. Si veda Gentile (2012), p. 69. 
molto intricato stabilire quale sia stato il ruolo assunto da Aristotele al suo interno. Alcuni platonici gli furono apertamente ostili (per esempio Attico, nel II d.C., frammenti del cui scritto contro Aristotele sono conservati nella Praeparatio Evangelica di Eusebio di Cesarea) ${ }^{23}$; altri però, pur criticandone alcune tesi, ritenevano che Aristotele facesse comunque parte della tradizione platonico-pitagorica. Fatto molto interessante, Plutarco di Cheronea difende questa posizione ed è plausibile che egli si rifacesse proprio a Eudoro di Alessandria, il quale si interessò agli scritti di Aristotele fornendone probabilmente una lettura pitagorizzante ${ }^{24}$.

Questa situazione si perpetua e, anzi, si accentua nel platonismo dopo Plotino, quando la lettura dei trattati aristotelici fu gradualmente integrata nel curriculum di studio neoplatonico. Le voci anche aspramente critiche verso Aristotele non mancarono (in particolare Siriano e lo stesso Proclo): Campanella è il loro ideale successore $^{25}$. Tuttavia, Aristotele fu di fatto integrato nel corpus dottrinale platonico e l'opera di commento dei neoplatonici sui trattati aristotelici, iniziata da Porfirio, ebbe un impatto decisivo sulle tradizioni filosofiche posteriori ${ }^{26}$. Alberto Magno e Tommaso d'Aquino, i maestri domenicani venerati da Campanella, sono profondamente debitori verso la lettura neoplatonizzante di Aristotele elaborata alla fine dell'antichità, lettura che essi avevano assimilato per lo più attraverso la mediazione degli $\mathrm{Arabi}^{27}$. La rinascita del platonismo ad opera di Ficino, il suo sforzo di mostrare l'unità tra le tradizioni teologiche antiche e la rivelazione biblica non segnano, dunque, una rottura completa rispetto alla tradizione precedente, ma il nuovo capitolo di una vicenda cominciata non con Pletone, Bessarione e Trapezunzio, ma ben quindici secoli prima, e mai veramente interrotta.

Campanella sintetizza in sé tutto l'intricato e lunghissimo sviluppo che lo ha preceduto. Il platonismo di ispirazione ficiniana lo collega direttamente alle fonti antiche che nella Philosophia sensibus demonstrata sono estesamente richiamate e

23 Su Attico, cfr. Baltes (1983) e Karamanolis (2006), pp. 150-190; edizione dei frammenti in des Places (1977). Sulla ricezione di Attico in Ficino, cfr. Monfasani (2002), pp. 180, 186.

${ }^{24}$ Cfr. Donini (1999).

25 Circa la posizione antiaristotelica di Siriano e Proclo, cfr. Steel (2003) e, da ultimo, HeLMIG (2012), pp. 205-208. L'ostilità programmatica verso Aristotele non impedisce però a questi autori di incorporare molte dottrine aristoteliche dentro il loro platonismo, particolarmente per quanto riguarda la concezione del mondo fisico: cfr. Opsomer (2009).

${ }^{26}$ La letteratura critica è, ancora un volta, molto vasta. In generale, sulla filosofia dei commentatori, si veda la ricca raccolta commentata di testi in SorabJi (2004). Una discussione critica di quest'opera, con alcune osservazioni sulla filosofia dei commentatori e la loro collocazione storica, si trova in Chiaradonna - Rashed (2010).

27 Su questo tema mi limito a rinviare a D’Ancona (1995). 
citate. Diversamente da molti esegeti neoplatonici, Campanella è antiaristotelico e la sua posizione è stata messa in parallelo con quella di altri critici rinascimentali di Aristotele, ad esempio Gianfrancesco Pico della Mirandola ${ }^{28}$. Ma, anche in questo caso, la situazione è più sfaccettata e sarebbe semplicistico equiparare l'atteggiamento di Campanella verso Aristotele a un semplice rifiuto. A caratterizzare la sua peculiare posizione è insieme il rifiuto di Aristotele e di Averroè, unito però all'ammirazione verso i maestri domenicani Alberto e Tommaso, le cui posizioni Campanella distanzia rispetto a quelle di Aristotele e dei Peripatetici. Campanella, insomma, si basa su Tommaso negando che egli sia un Peripatetico ${ }^{29}$. Ciò spiega perché, se non ci si ferma alle enunciazioni programmatiche ma si seguono le argomentazioni di Campanella, si osserva come l'eredità aristotelica sia in realtà ben presente. Più precisamente, come vedremo in seguito, si può trovare in Campanella la presenza di temi metafisici aristotelici letti alla luce della dottrina platonica o platonizzante della gerarchia metafisica e della partecipazione. Ancora una volta le origini ultime di questa posizione vanno reperite nella tarda antichità, ossia nell'Aristotele trasformato e platonizzato dei commentatori neoplatonici ${ }^{30}$. Certamente non antica è, invece, la nuova astronomia che, come vedremo, per Campanella scardina definitivamente il quadro aristotelico del cosmo. Ma proprio su questo punto la sua complessa collocazione storica emerge nel modo più evidente. Campanella, infatti, difende la nuova scienza dentro un quadro concettuale ancora in gran parte antico. A testimoniarlo è proprio la sua opera innovatrice per eccellenza, ossia l'Apologia pro Galileo, nella quale Galileo è a sua volta iscritto nella tradizione della prisca theologia posta sotto il patrocinio di Mosè e Pitagora.

\section{Il dibattito sul Timeo}

È opportuno tornare, adesso, alla sezione della Philosophia sensibus demonstrata da cui ha preso le mosse il presente contributo. Campanella non soltanto richiama la tesi degli antichi divinissimi viri della prisca theologia, ma aderisce a una precisa lettura del Timeo di Platone. Si tratta dell'esegesi letterale del dialogo, secondo la quale il Demiurgo ha realmente prodotto il cosmo e lo preserva dalla distruzione. Nell'antichità questa lettura del dialogo si opponeva a quella metaforica, secondo

\footnotetext{
${ }^{28}$ Così Mahoney (2003), pp. 26-32. Si veda anche Muccillo (2012).

${ }_{29}$ Fondamentali, per ricostruire la posizione di Campanella a questo riguardo, sono le sezioni dedicate a Tommaso nel De gentilismo non retinendo. Cfr. Campanella (1636), pp. 36-41 («Iuxta D. Thomam Aristoteles non est optimus philosophorum [...]»), 41-47 («Divus Thomas non est Peripateticus»).

30 Cfr. Chiaradonna (2010), pp. 330-331.
} 
cui la generazione descritta da Platone sarebbe solo l'espediente pedagogico per illustrare la dipendenza eterna del cosmo dal suo principio divino. Aristotele, nel criticare Platone (De caelo, I, 10-12), fa propria la lettura letterale del dialogo che fu difesa da una minoranza di Platonici nell'antichità, segnatamente Plutarco e Attico (I-II d.C.). Maggioritaria (anche in considerazione delle critiche mosse da Aristotele verso l'interpretazione letterale) risultò invece l'esegesi metaforica del dialogo e, in particolare, del verbo gegone con cui Platone (Timaeus 28b) descrive la generazione del mondo. Tutti i neoplatonici da Plotino in poi ritennero che il cosmo fosse eterno e dipendesse eternamente da principi superiori: il Timeo esprimerebbe in modo didattico questa relazione di dipendenza metafisica ${ }^{31}$.

Campanella è al corrente di questi dibattiti, attraverso Marsilio Ficino, e li riassume aderendo ancora una volta alla posizione della prisca theologia: egli si esprime dunque a favore dell'esegesi letterale del Timeo, ponendone in evidenza la convergenza rispetto alla rivelazione biblica e fondando l'accordo tra Mosè e Platone non solo su Ficino, ma sull'autorità antica del medioplatonico Numenio di Apamea, secondo il quale Platone non sarebbe altro se non Mosè che parla attico ${ }^{32}$. Il passo è molto interessante perché sintetizza in una sola pagina circa 2000 anni di riflessione cosmologica:

Verum Platonicorum secta in contrarias opiniones de Platonis intentione scinditur. Crantor enim, Plotinus, Porphyrius, Proclus et Iamblychus $\mathrm{ab}$ aeterno effectum fuisse a Deo contendunt; et cum dicit Plato mundum praecessisse inordinatum et confusum, deinde redactum in ordinem, sumunt hunc ordinem sequi secundum modum intelligendi, et prioritatem naturae, non temporis concedunt. Severus autem, Atticus, Plutarchus, Marsilius ac alii multi in temporis principio de novo factum fuisse a Deo demonstrant in via Platonis; quibus assentiendum magis censeo; Plato enim, ut Numenius Platonicus inquit, Atticus est Moyses nil ab eo discrepans. [... $]^{33}$.

L'attacco ad Aristotele segue immediatamente e il tono è quello, consueto, dell'invettiva. Aristotele ha negato la libertà di Dio e l'esistenza stessa della provvi-

31 Sulla generazione del cosmo nel Timeo nell'esegesi antica, si veda l'ottima rassegna commentata di testi in DörRIE ( $\dagger$ ) - BALTEs (1998), pp. 84-146 (testi) and 373-465 (commento).

32 Cfr. Numenio, fr. 8 des Places (il detto è riportato da Eusebio di Cesarea, Praeparatio Evangelica, IX, 6, 9; XI, 10, 14 e Clemente Alessandrino, Stromata, I, 22, 150, 4).

33 Campanella (1992), p. 286. 
denza $^{34}$. Il suo Dio non provvede a nulla, se non ad assicurare un moto eterno coevo al mondo eterno; non pensa a nulla se non a sé stesso. Per queste sue opinioni, osserva Campanella, a buon diritto i Greci condannarono Aristotele per empietà e lo costrinsero a rifugiarsi a Calcide ${ }^{35}$. Nella Philosophia sensibus demonstrata, però, Campanella non si dilunga sulla confutazione filosofica di Aristotele. Si limita a richiamare al-Ghazali e Giovanni Filopono, che hanno respinto la concezione eternalistica di Aristotele in via naturali: dopo di loro, ulteriori difese della tesi creazionistica appaiono superflue (frivolae). In breve: la confutazione filosofica di Filopono e al-Ghazali, unita a quella della teologia cristiana, mostra a sufficienza l'errore di Aristotele. Campanella non approfondisce la questione e passa ad argomentare a favore della costituzione ignea del cielo.

\section{L'eredità degli Antichi: esegesi, commenti e prisca theologia}

Come L'agenda stabilita nella Philosophia sensibus demonstrata rimane costante nelle opere più tarde. Per il loro carattere programmatico è importante richiamare almeno i punti contro Aristotele formulati nell'Apologia pro Galileo (1616), un quarto di secolo dopo ${ }^{36}$. Come è prevedibile, le autorità citate da Campanella sono, questa volta, patristiche: egli sostiene che, se si condanna Galileo per essersi opposto ad Aristotele, si dovranno condannare anche i Padri che hanno negato le concezioni peripatetiche, ossia Ambrogio, Basilio, Eusebio, Origene, Crisostomo, Giustino e gli altri santi Dottori della Chiesa che hanno rifiutato i principi aristotelici non soltanto nella metafisica, ma anche nella fisica, accostandosi piuttosto a Platone e agli Stoici ${ }^{37}$. Ecco l'elenco delle «eresie infestanti» di Aristotele ${ }^{38}$ :

${ }^{34}$ L'obiezione è tradizionale e si trova già formulata in Attico, il quale su questo punto metteva in parallelo polemicamente Aristotele ed Epicuro: cfr. Eusebio di Cesarea, Praeparatio Evangelica, XV, 5, 1-14 = Attico, fr. 3 des Places.

35 Cfr. Campanella (1992), p. 287.

36 Nelle critiche rivolte contro Aristotele, l'Apologia è assai vicina allo scritto De gentilismo non retinendo (cfr. supra, n. 2). Si vedano le ossevazioni di M.-P. Lerner in Campanella (2006), pp. XXXV-XXXVI. Si è però preferito considerare l'Apologia, perché consente di cogliere meglio il legame tra la critica di Aristotele e l'elaborazione di una nuova cosmologia in Campanella.

37 Cfr. Campanella (2006), pp. 106-108. Il ricco apparato di note curato da M.-P. Lerner permette di rintracciare le fonti su cui si fonda Campanella.

38 Cfr. Campanella (2006), pp. 108-109. Una lista parallela si trova del De gentilismo: cfr. Campanella (1636), pp. 16-18. 
1: Eternità del movimento, affermata da Aristotele nel libro VIII della Fisica e nel XII della Metafisica ${ }^{39}$.

2: Mortalità dell'anima (o meglio: esiste un'unica anima immortale comune a tutti gli uomini).

3: Dio non si cura di quanto accade nel mondo inferiore.

4: Dio imprime un moto contrario a quello degli angeli.

5: gli inferi sono una favola.

6: Dio agisce in modo necessario

7: il caso elimina la provvidenza.

I punti che riguardano la cosmologia (eternità del moto, negazione della provvidenza e della libertà divina) sono gli stessi già presenti nella Philosophia sensibus demonstrata. Diverso, ma analogo, è d'altronde lo scopo di Campanella: nella sua opera giovanile si tratta di difendere Telesio dalle imputazioni degli aristotelici ritorcendo contro di essi le accuse di eresia; nell'Apologia pro Galileo si tratta di difendere Galileo da imputazioni analoghe, ritorcendo ancora una volta contro i Peripatetici le accuse di eresia. Le tesi aristoteliche contestate non cambiano.

Se però si vuol entrare nel dettaglio delle argomentazioni usate da Campanella, l'opera di riferimento è senza dubbio la Metaphysica. La sua composizione occupò, in varie stesure, l'intera vita di Campanella e il testo venne finalmente pubblicato a Parigi nel 1638, solo tre anni prima delle Meditationes de prima philosophia di Descartes ${ }^{40}$. La Metaphysica di Campanella è un'opera stupefacente, autentica summa della metafisica "pre-moderna». Vi confluisce, in una sintesi sterminata, tutta la tradizione dai Presocratici al Cinquecento, passando per Platone, Aristotele, le scuole ellenistiche e il neoplatonismo, i Padri della Chiesa — dei quali Campanella possiede una conoscenza estesissima— gli Arabi, i Maestri medievali, Ficino ... Una sintesi per certi aspetti prodigiosa, per

39 Cfr. Aristotele, Physica, VIII, 1-2, 250b10-253a21; 7, 261 a23 ss.; Metaphysica, XII, 6, 1071b5-12; 7, 1072a21 ss.

${ }^{40}$ Sulle diverse redazioni della Metaphysica, cfr. Firpo (1940), pp. 96-99. 
altri persino inquietante di 2000 anni di filosofia. Piuttosto paradossalmente, la Metaphysica è però ancora oggi abbastanza poco nota e molte sezioni rimangono del tutto inesplorate.

Un intero libro, l'undicesimo, ha per titolo De mundi aeternitate, principio, et fine et aetate et mutatione sententia diversa. I temi tracciati altrove vi trovano pieno sviluppo. L'argomentazione è dettagliata, se non prolissa, e non è possibile ripercorrerla nel dettaglio qui. Per la presente discussione è sufficiente soffermarsi sulla sezione in cui Campanella illustra e confuta gli argomenti peripatetici a favore dell'eternità del mondo ${ }^{41}$. I bersagli polemici sono Aristotele e Ocello Lucano, ossia l'autore del trattato pseudopitagorico peripatetizzante De universi natura (Peri tas tô pantos physios) attribuito per l'appunto a Ocello Lucano e ora datato dagli studiosi intorno al 150 a.C. ${ }^{42}$. Esattamente come nell'Apologia pro Galileo, Campanella richiama (parafrasando liberamente) gli argomenti di Physica, VIII e Metaphysica, XII sull'eternità del movimento. Sempre riguardo all'eternità del moto, segue un importante richiamo a De caelo, I, 3, 270b11-16, dove Aristotele afferma che le osservazioni astronomiche confermano il carattere inalterabile del cielo. Ancora una volta, la parafrasi è assai libera ed espansa rispetto al testo aristotelico, giacché Campanella vi unisce il resoconto di Diodoro Siculo sulle osservazioni dei Caldei (II, 30):

Probat rursus motus perpetuitatem in primo libro De caelo, eo quod Chaldaei, qui per saecula multa syderum motum observarunt, et ut Diodorus ait, per 403.000 semper in eodem situ ipsa posuerunt, et mensura motus nulla irregularitate facta in velocius, aut tardius, vel in alia Caeli latitudine, et plaga, neque diversificato unquam motu recto vel obliquo: ergo omnia perseverant sicut ab aeterno creaturae, et stultus est timor Empedoclis et aliorum, qui Mundi interitum expectant ${ }^{43}$.

L'argomento che dimostra l'incorruttibilità del mondo a partire dalle osservazioni astronomiche è tradizionale ed ebbe notevole fortuna ${ }^{44}$. Tra gli argomenti peripatetici che seguono nella lista di Campanella, vi sono quello

41 Cfr. Campanella (1638), pars III, XI, 3, pp. 10-18.

42 Sulla sua presenza in Campanella, cfr. la nota di De Franco in CAmpanella (1992), p. 20 n. 50. Lo scritto era stato tradotto in latino da Ludovico Nogarola e pubblicato a Venezia nel 1559. Edizione del testo greco in Harder (1926); si veda Moraux (1984), pp. 635-637.

43 Campanella (1638), pars III, XI, 3, p. 10.

44 Si trovava, ad esempio, nel perduto De demonstratione di Galeno, come testimonia il medico e filosofo arabo al-Razi nell'opera Dubbi contro Galeno (3.18-21 Mohaghegh; traduzione 
sull'incorruttibilità degli elementi e quello che dimostra l'eternità del cosmo in base alla natura essenzialmente e necessariamente incorruttibile del cielo: Dio stesso non può alterarla. Campanella fa esplicito riferimento ad Alessandro di Afrodisia, il quale effettivamente sostenne questa posizione, forse rivolgendosi contro Attico e Galeno i quali ritenevano che il mondo potesse essere incorruttibile perché costantemente preservato da una causa esterna (Dio ${ }^{45}$. Dalla tesi peripatetica consegue che il mondo è necessariamente eterno e Dio non può in nessun modo intervenire sulla sua generazione e corruzione.

Subito dopo aver esposto gli argomenti peripatetici, Campanella ribadisce lapidariamente la propria posizione creazionistica che Miguel Ángel Granada ha efficacemente definito una "cosmologia escatologica» ${ }^{46}$. In accordo a essa il mondo è in progressiva evoluzione verso la propria fine, nella quale la Terrà sarà consumata dal fuoco a causa dell'inevitabile avvicinamento del sole:

Nos autem credimus, Mundum esse factum, et non casu quodam, nec necessitate cogente, sed a Potentissimo, Sapientissimo, et optimo Factore, non ab aeterno, sed noviter: et non ex materia praecedenti, neque ex ruinis alterius mundi, sed ex nihilo. Et dissolvendum esse aliquando, sed non in nihilum, sed in meliorem formam instaurandum; nec dum enim ad sui perfectionem devenit ${ }^{47}$.

Negli argomenti che seguono, emergono, accanto a motivi ben noti, come la confutazione della dottrina del quinto elemento e l'affermazione della natura ignea del cielo, due cruciali elementi dottrinali che caratterizzano la Metaphysica: da un lato la concezione metafisica sul rapporto tra Dio e il cosmo, dall'altro il significato delle nuove osservazioni astronomiche. La dottrina della partecipazio-

inglese in McGinnis-Reisman (2007), pp. 49-53); cfr. anche Simplicio, In De Caelo, 117, 20-31 Heiberg.

45 Campanella (1638), pars III, XI, 3, p. 11: «Item mundus est per se incorruptibilis secundum Aristotelem sicut triangulus per se habet tres, et necessario; ergo non indiget extrinseco conservatore: sed est Deus, sicut pars eius. Unde Aphrodiseus.q.nat.lib.2.c. 19 ostendit, Mundum non indigere Deo provisore, ut sit, neque ut bene sit, [...] ergo mundi essentia non indiget Deo [...]». Il riferimento è ad Alessandro di Afrodisia, Quaestiones, II, 19. La critica delle tesi di Alessandro di Afrodisia non è inusuale e compare già nell'Adversus calumniatorem Platonis di Bessarione (II, IX, 7): cfr. Bessarione (2013), p. 74.

46 Cfr. Granada (2007), p. 285.

47 Campanella (1638), pars III, XI, 3, p. 11. Sul rapporto tra tempo e creazione del mondo in Campanella, cfr. Ponzio (2010), p. 370-372, che sottolinea gli aspetti antiaristotelici della concezione campanelliana. 
ne collega direttamente Campanella alla tradizione neoplatonica, trasposta però in un modello creazionistico secondo cui la distinzione Dio / mondo divide un agente infinito rispetto a un prodotto finito che ha l'essere solo per partecipazione:

Item ostensum est in superioribus sermonibus esse principium infinitum Ens per essentiam causam omnis finiti Entis, et per participationem: cur ergo Mundus finitus sit, et Ens per participationem, semper esse non potuit nisi factus ab illo primo $[\ldots]^{48}$.

È l'esatto rovesciamento dell'argomento di Alessandro di Afrodisia, per cui il mondo è essenzialmente incorruttibile e Dio non può in alcun modo intervenire sulla sua generazione e corruzione. Per Campanella, invece, la dicotomia tra Dio infinito e mondo finito impedisce di porre il mondo come eterno. Il mondo non può esistere senza Dio, mentre Dio può esistere senza il mondo. Questo, dunque, non si muove necessariamente ab aeterno, come erroneamente supposto da Aristotele.

La cesura tra l'essere infinito di Dio e l'essere finito del mondo conduce Campanella a respingere la celebre analogia dei Platonici, riportata da Agostino secondo cui il mondo avrebbe avuto un inizio solo nel senso della dipendenza causale e non della creazione, proprio come se un piede fosse impresso ab aeterno nella polvere e l'impronta vi fosse eternamente presente ${ }^{49}$. Campanella ribadisce lo scarto metafisico tra causa e causato che impedisce di porli come coevi. L'impronta non è in alcun modo eternamente congiunta a Dio. Con la sua tipica e immaginosa terminologia filosofica, Campanella afferma l'assoluta distinzione tra l'essenza infinita di Dio e la natura finita e partecipata del mondo creato, al quale sono propri il tempo e il non essere:

Mundus enim participat non Esse et tempus; nec toticipat Esse. Ergo non habet totum esse, ergo neque aeternitatem. Siquidem probatum est a nobis, tempus esse, non entis prolem et impotentiam simul existendi totaliter totum $^{50}$.

48 Campanella (1638), pars III, XI, 3, p. 12.

49 Cfr. Agostino, De Civitate Dei, X, 31.

50 Cfr. Campanella (1638), pars III, XI, 3, p. 13. Sulla terminologia metafisica di Campanella, si veda Giglioni (2010). 


\section{Eternità del mondo e nuova astronomia: Campanella tra Antico e Moderno}

Fin qui la posizione di Campanella ricalca temi ben noti e antichi di secoli. La sua è una elaborata versione del neoplatonismo nella quale la metafisica della partecipazione è adattata ai principi della teologia creazionistica. Tuttavia, la confutazione di Aristotele non si svolge soltanto secondo queste linee, ma integra anche le osservazioni astronomiche che mutano profondamente il quadro fatto proprio dai Peripatetici. Sono i nuovi dati che permettono di confutare il canonico argomento basato sull'osservazione dell'uniformità del cielo. Le osservazioni, in realtà, dimostrano tutt'altro: "Quod autem dissolvendus sit mundus per ignem, non modo fides testatur omnium nationum, sed et ipsarum rerum primarum mutata continuo facies $[\ldots] »^{51}$. Le conclusioni dei Caldei sono dunque erronee e le costellazioni hanno in realtà mutato posizione: «ostensum est [...] situm siderum non eundem $»^{52}$.

A questo punto, è inevitabile tornare all'Apologia pro Galileo e al celebre richiamo di Campanella alle osservazioni di Tycho Brahe e Galileo, a cominciare dall'apparizione della nuova stella nella costellazione di Cassiopea nel 1572, che scardinano definitivamente l'orizzonte della cosmologia tradizionale e mettono in questione gli stessi principi dell'astronomia accettati da Campanella circa la composizione ignea del cielo. Questa volta la confutazione della composizione eterea del cielo e dell'eternalismo aristotelico attinge direttamente alla nuova scienza:

Similiter facta est anno 1572 stella absque parallaxi in sedili Cassiopeae nova ex vaporibus, ut Tycho et innumeri mathematici scripserunt, qui viderunt et observarunt: ergo sunt vapores in stellis. Item cometae fiunt supra lunam, ut probant instrumenta, quod Aristoteles negavit ${ }^{53}$.

51 Campanella (1638), pars III, XI, 3, p. 13.

52 Campanella (1638), pars III, XI, 3, p. 15.

53 Campanella (2006), p. 168 e cfr. ivi, p. 60. Come osserva M.-P. Lerner, «[n]el mondo sopralunare di Aristotele non c'è posto per fenomeni come le nuvole solari in costante cambiamento, né per la nascita e la morte di astri come le novae del 1572 e del 1604, né per la generazione sopra la luna di corpi effimeri come le comete» [in CAMPANella (2006), p. 60 n. 77]. Circa la ricezione degli Astronomiae instauratae progymnasmata di Brahe in Campanella, cfr. anche Granada (2007), p. 283. 
$\grave{E}$, certamente, su questo preciso punto che la critica di Campanella ad Aristotele si allontana dallo schema, antico di secoli, della prisca theologia e dei suoi diversi orientamenti. L'eternalismo peripatetico e la dottrina dell'uniformità e inalterabilità del cielo sono irrimediabilmente confutati dalle nuove osservazioni. In questo modo Campanella non si rivela dunque solo l'ultimo degli antichi, ma diventa alfiere della nuova astronomia con cui comincia la scienza moderna.

Tutto ciò è innegabile, ma ancora una volta il quadro si rivela difficile da valutare $^{54}$. A mostrarlo è la stessa Apologia, nella quale l'innovazione è insieme celebrata e inserita in un quadro di pensiero antichissimo. Si può così comprendere perché Campanella, con una vera acrobazia, non solo insista sul motivo convenzionale del pitagorismo di Copernico, ma addirittura inserisca Galileo nello schema della prisca theologia che a suo dire comprende Mosè e Pitagora, e fu combattuta da Aristotele:

Praeterea hanc sententiam Galilei esse vetustissimam, tam de motu terrae, quam de solis in centro mansione, et de systematis sydereis et aquis et elementis eorum, in fine docebimus, imo ab ipso Moyse ortam esse: etiam Pythagoram, genere Iudaeum, licet in Graeciae natum urbe, teste $S$. Ambrosio, in Italiam attulisse ipsam, et in Graeciam, et Crotone Calabrorum docuisse, ac inanibus rationibus ab Aristotele impugnatam esse, absque mathematica demonstratione, ex quadam morali ac rustica coniectura ${ }^{55}$.

Nel suo importante commento allo scritto campanelliano Michel-Pierre Lerner interpreta molto sottilmente l'argomentazione polemica di Campanella:

Il vantaggio che Campanella trae da questa curiosa filiazione dottrinale sul piano della sua argomentazione è immediatamente percepibile. Da un lato, pur essendo un moderno, Galileo non è un 'novatore' nel senso negativo conferito usualmente a questo termine, poiché si limita a riprendere gli insegnamenti di Copernico, essi stessi derivati dalle dottrine pitagoriche. Dall'altro, ritrovando una tradizione sacra sulla strut-

${ }^{54}$ Non ci sono elementi sufficienti, d'altronde, per stabilire che Campanella aderì al copernicanesimo e l'Apologia pro Galileo non intendeva tanto sostenere il copernicanesimo, quanto difendere la libertas philosophandi dimostrando che il modo di filosofare proprio di Galileo era più consono alle scritture rispetto a quello aristotelico. Si veda Granada (2007), p. 283.

55 Campanella (2006), p. 30; si veda anche ivi, p. 190. 
tura dell'universo la cui verità tende a imporsi con le recenti scoperte astronomiche, il cristiano Galileo restituisce all'Italia, a Mosè e a Roma la loro legittima proprietà e difende la filosofia sacra di Mosè contro i danni causati dai pagani. Invocando questo nesso tra Mosè e Pitagora, Campanella spera di convincere i suoi lettori a respingere la loro paralizzante infeudazione ad Aristotele, una tutela che ha come conseguenza quella di mascherare l'autentico rapporto tra i Moderni e gli Antichi, vale a dire la superiorità dei primi sui secondi $[\ldots]^{56}$.

Questa penetrante analisi è senza dubbio condivisibile nelle sue conclusioni generali, ma rischia di ridurre il richiamo alla prisca theologia a poco più di un espediente retorico usato da Campanella per rendere accettabile la nuova astronomia. Tuttavia una simile lettura finirebbe per non tener conto del ruolo assolutamente centrale che, come si è visto, svolge nel suo pensiero la concezione della tradizione filosofica e teologica originata nel neoplatonismo antico e perpetuatasi fino al Rinascimento. Forse il richiamo a Mosè e a Pitagora, proprio nello scritto più impegnato di Campanella a favore della nuova astronomia, testimonia della sua collocazione storica, nella quale l'antico e il moderno si intrecciano indissolubilmente.

\section{Ringraziamenti}

Desidero ringraziare vivamente Germana Ernst, che ha letto una prima versione di questo studio, e Miguel Ángel Granada, per i suoi preziosi suggerimenti. Sono, naturalmente, il solo responsabile di eventuali errori.

\section{Bibliografía}

\section{Opere di Campanella}

Campanella (1636). Tommaso Campanella, De gentilismo in retinendo, in Atheismus Triumphatus, seu Contra Antichristianismum; De gentilismo non retinendo; De Praedestinatione et Reprobatione et auxiliis divinae gratiae Cento Thomisticus, Apud T. Dubray, Parisiis, pp. 1-63.

Online: http://www.iliesi.cnr.it/Campanella/testi_sql.php?lk=Ath\&p=1nna\&pt=1\&x=1

56 M.-P. Lerner in Campanella (2006), p. XLIII. 
- (1638). Tommaso Campanella, Metaphysica, Parisiis (ristampa anastatica a cura di L. Firpo, Bottega d'Erasmo, Torino 1961).

Online: http://www.iliesi.cnr.it/Campanella/testi_sql.php?lk=Met\&p=I1nn\&pt=

- (1992). Tommaso Campanella, Philosophia sensibus demonstrata, a cura di Luigi De Franco, Vivarium, Napoli.

- (2006). Tommaso Campanella, Apologia pro Galileo, a cura di Michel-Pierre Lerner, traduzione di Germana Ernst, Edizioni della Normale, Pisa.

\section{Opere di altri autori}

Bessarione (2013). Basilio Bessarione, Contro il calunniatore di Platone, introduzione, traduzione e cura di Eva Del Soldato, nota critica di Ivanoe Privitera, Edizioni di Storia e Letteratura, Roma.

Ficino (1576). Marsilio Ficino, Opera omnia, Ex officina Henricpetrina, Basileae.

- (2011). Mercurii Trismegisti Pimander sive liber de potestate et sapientia Dei, a cura di Maurizio Campanelli, Aragno, Torino.

\section{Studi}

Baltes (1972). Matthias Baltes, Timaios Lokros. Über die Natur des Kosmos und der Seele. Kommentiert von M. B., Brill, Leiden.

- (1983). Matthias Baltes, «Zur Philosophie des Platonikers Attikos», in H.-D. Blume - F. Mann, eds., Platonismus und Christentum. Festschrift für Heinrich Dörrie, Aschendorff, Münster (rist. in M. Baltes, Dianoêmata. Kleine Schriften zu Platon und zum Platonismus, Teubner, Stuttgart - Leipzig 1999, pp. 81-111).

Bonazzi - Opsomer (2009). Mauro Bonazzi - Jan Opsomer, eds., The Origins of the Platonic System. Platonisms of the Early Empire and their Philosophical Contexts, Peeters, Leuven 2009.

Centrone (1990). Bruno Centrone, Pseudopythagorica ethica, I trattati morali di Archita, Metopo, Teage, Eurifamo, Bibliopolis, Napoli.

Chiaradonna (2010). Riccardo Chiaradonna, s.v. «Plotino (Plotinus)», E. Canone G. Ernst, eds., Enciclopedia Bruniana e Campanelliana, vol. II, Serra, Pisa-Roma, pp. 322-332.

Chiaradonna-Rashed (2010). Riccardo Chiaradonna - Marwan Rashed, «Before and After the Commentators: An Exercise in Periodization. A discussion of Richard Sorabji, The Philosophy of the Commentators, 200-600 AD", Oxford Studies in Ancient Philosophy, 38, pp. 251-297. 
D’Ancona (1995). Cristina D’Ancona, Recherches sur le Liber de Causis, Vrin, Paris.

Del Soldato (2012). Eva Del Soldato, «Illa litteris Graecis abdita: Bessarion, Plato, and the Western World", in M. Sgarbi, ed., Translatio Studiorum. Ancient, Medieval, and Modern Bearers of Intellectual History, Brill, Leiden, pp. 109-122.

Des Places (1973). Édouard des Places, Numénius: Fragments. Texte établi et traduit, Les Belles Lettres, Paris.

- (1977). Édouard des Places, Atticus: Fragments. Texte établi et traduit, Les Belles Lettres, Paris.

Diels (1879). Hermann Diels, Doxographi Graeci. Collegit, recensuit, prolegomenis indicibusque instruxit H. D., Reimer, Berlin.

Donini (1994). Pierluigi Donini, «Testi e commenti, manuali e insegnamento: la forma sistematica e i metodi della filosofia in età postellenistica», in Aufstieg und Niedergang der römischen Welt, II, 36, 7, de Gruyter, Berlin - New York, pp. 5027-5100 (rist. in P. Donini, Commentary and Tradition. Studies in Aristotelianism, Platonism, and Post-Hellenistic Philosophy, ed. by M. Bonazzi, de Gruyter, Berlin - New York 2011, pp. 211-281).

- (1999). Pierluigi Donini, «Platone e Aristotele nella tradizione pitagorica secondo Plutarco", in A. Pérez Jiménez - J. García López - R. Ma Aguilar, eds., Plutarco, Platón y Aristóteles, Actas del V Congreso Internacional de la I.P.S., Ediciones Clásicas, Madrid, pp. 9-24 (rist. in P. Donini, Commentary and Tradition. Studies in Aristotelianism, Platonism, and Post-Hellenistic Philosophy, ed. by M. Bonazzi, de Gruyter, Berlin - New York 2011, pp. 359-373).

Dörrie $(\dagger)$ - Baltes (1998). Heinrich Dörrie $(\dagger)$ - Matthias Baltes, Der Platonismus in der Antike, vol. 5 (Bausteine 125-150): Die philosophische Lehre des Platonismus (2), Frommann-Holzboog, Stuttgart - Bad Cannstatt.

ERnst (2002). Germana Ernst, Tommaso Campanella. Il libro e il corpo della natura, Laterza, Roma - Bari.

Firpo (1940). Luigi Firpo, Bibliografia degli scritti di Tommaso Campanella. Pubblicazione promossa dalla R. Accademia delle scienze di Torino nel III centenario della morte di T. Campanella, Bona, Torino.

Online: http://www.iliesi.cnr.it/Campanella/pdf/FirBF.pdf

Frede (1999). Michael Frede, «Epilogue», in K. Algra - J. Barnes - J. Mansfeld - M. Schofield, eds., The Cambridge History of Hellenistic Philosophy, Cambridge University Press, Cambridge.

GARIN (1975). Eugenio Garin, Rinascite e rivoluzioni: movimenti culturali dal XIV al XVII secolo, Laterza, Bari. 
GatTi (2010). Paola Gatti, Il gran libro del mondo nella filosofia di Tommaso Campanella, Gregorian \& Biblical Press, Roma.

Gentile (2012). Sebastiano Gentile, "Considerazioni intorno al Ficino e alla Prisca Theologia», in S. Caroti - V. Perrone Compagni, eds., Nuovi Maestri e Antichi Testi. Umanesimo e Rinascimento alle origini del pensiero moderno, Atti del convegno internazionale di studi in onore di Cesare Vasoli, Olschki, Firenze, pp. 57-72.

Giglioni (2010). Guido Giglioni, s.v. «Primalità (primalitas)», in E. Canone - G. Ernst, eds., Enciclopedia Bruniana e Campanelliana, vol. II, Serra, Pisa-Roma, pp. 332-348.

Granada (2007). Miguel Ángel Granada, "New Visions of The Cosmos», in J. Hankins, ed., The Cambridge Companion to Renaissance Philosophy, Cambridge University Press, Cambridge, pp. 270-286.

Надот (1987). Pierre Hadot, «Théologie, exégèse, révélation, écriture dans la philosophie grecque», in M. Tardieu, ed., Les règles de l'interprétation, Cerf, Paris 1987, pp. 13-34 (rist. in P. Hadot, Études de philosophie ancienne, Les Belles Lettres, Paris 1998, pp. 27-58).

Hankins (1990). James Hankins, Plato in the Italian Renaissance, 2 voll. (numerazione delle pagine continua), Brill, Leiden.

- (2000). James Hankins, "Galileo, Ficino and Renaissance Platonism», in J. Kraye M.W.F. Stone, eds., Humanism and Early Modern Philosophy, London, Routledge, pp. 208-237.

Harder (1926). Richard Harder, Ocellus Lucanus. Text und Kommentar, Weidmann, Berlin.

Headley (1990). John M. Headley, «Tommaso Campanella and Jean de Launoy: The Controversy over Aristotle and his Reception in the West», Renaissance Quarterly, 43, pp. 529-550.

Helmig (2012). Christoph Helmig, Forms and Concepts: Concept Formation in the Platonic Tradition, de Gruyter, Berlin - Boston.

Karamanolis (2006). G. Karamanolis, Plato and Aristotle in Agreement? Platonists on Aristotle from Antiochus to Porphyry, Clarendon Press, Oxford 2006.

Mahoney (2003). Edward P. Mahoney, «Aristotle and Some Late Medieval and Renaissance Philosophers», in R. Pozzo, ed., The Impact of Aristotelianism on Modern Philosophy, The Catholic University of America Press, Washington DC, pp. 1-34.

Mansfeld - Runia (1997). Jaap Mansfeld - David Runia, Aëtiana: The Method and Intellectual Context of a Doxographer, vol. 1: The Sources, Brill, Leiden.

McGinnis - Reisman (2007), Jon McGinnis - David C. Reisman, Classical Arabic Philosophy: An Anthology of Sources, Hackett, Indianapolis - Cambridge. 
Monfasani (2002). John Monfasani, «Marsilio Ficino and the Plato-Aristotle Controversy", in M.J.B. Allen - V. Rees with M. Davies, eds., Marsilio Ficino: His Philosophy, his Theology, his Legacy, Brill, Leiden, pp. 179-202.

Moraux (1984). Paul Moraux, Der Aristotelismus bei den Griechen von Andronikos bis Alexander von Aphrodisias, vol. 2: Der Aristotelismus im i. und ii. Jh.n. Chr., de Gruyter, Berlin - New York.

Muccillo (2012). Maria Muccillo, «Platonismo e anti platonismo nel tardo Rinascimento: echi di dottrine patriziane negli scritti di Paolo Beni e Giovanni Battista Crispo di Gallipoli», in S. Caroti - V. Perrone Compagni, eds., Nuovi Maestri e Antichi Testi. Umanesimo e Rinascimento alle origini del pensiero moderno, Atti del convegno internazionale di studi in onore di Cesare Vasoli, Olschki, Firenze, pp. 351-399.

Opsomer (2009). Jan Opsomer, "The Integration of Aristotelian Physics in a Neoplatonic Context: Proclus on Movers and Divisibility», in R. Chiaradonna - F. Trabattoni, eds., Physics and Philosophy of Nature in Greek Neoplatonism, Leiden, Brill, pp. 189-230.

Ponzio (2010). Paolo Ponzio, s.v. «Tempo (tempus)», in E. Canone - G. Ernst, eds., Enciclopedia bruniana e campanelliana, vol. II, Serra, Pisa - Roma, pp. 369-377.

SAffrey (1992). Henri Dominique Saffrey, «Accorder entre elles les traditions théologiques: une caractéristique du néoplatonisme athénien», in E.P. Bos - P.A. Meijer, eds., On Proclus and his Influence in Medieval Philosophy, Brill, Leiden, pp. 35-50 (rist. in H.D. Saffrey, Le Néoplatonisme après Plotin, Vrin, Paris 2000, pp. 143-158).

Schofield (2013). Malcolm Schofield, ed., Aristotle, Plato and Pythagoreanism in the First Century BC: New Directions for Philosophy, Cambridge University Press, Cambridge.

SEdley (2003). David Sedley, "Philodemus and the Decentralisation of Philosophy», Cronache ercolanesi, 33, pp. 31-41.

- (2012). David Sedley, ed., The Philosophy of Antiochus, Cambridge University Press, Cambridge.

Sorabji (2004). Richard Sorabji, The Philosophy of the Commentators, 200-600 AD: A Sourcebook, 3 voll., Cornell University Press, Ithaca NY.

Steel (2003). Carlos Steel, «Why Should We Prefer Plato's Timaeus to Aristotle’s Physics? Proclus' Critique of Aristotle's Causal Explanation of the Physical World», in R.W. Sharples - A. Sheppard, eds., Ancient Approaches to Plato's Timaeus, BICS Suppl. 78, Institute of Classical Studies, London, pp. 175-187. 
Taormina (2012). Daniela Patrizia Taormina, «Platonismo e pitagorismo», in R. Chiaradonna, ed., Filosofia tardoantica. Storia e problemi, Carocci, Roma, pp. 103-127.

Recibido: 17/12/2013

Aceptado: 10/01/2014 
